\title{
Natural Disasters in Europe within Years 2011-2020
}

\author{
Marek Hoffmann \\ Faculty of Civil Engineering, Department of Geomatics, Czech Technical University in Prague - marek.hoffmann1212@gmail.com
}

Keywords: Thematic maps, Hex map, Natural Disasters

\begin{abstract}
:
This map serves as a poster of one map that consists of several cartographic methods combined. The map tracks natural disasters that occurred in the area of the European continent within the last decade. It was made as a semestral project of "Projekt kartografie" course at Czech Technical University in Prague within a Geomatics Master's degree study program.

Data were acquired by the query select from the Emergency Event Database (EM-DAT) that contains data on the occurrence and effects of disasters in the world from 1900 until today. EM-DAT was launched by the Centre for Research on the Epidemiology of Disasters with the initial support of the World Health Organisation and the Belgian Government. Its main purpose is to rationalize decision-making and to determine priorities in case of abrupt disaster occurrence. Polygons of countries were gathered from the European Statistical Office (Eurostat) on a scale of 1:10 million matching the intended map appearance.
\end{abstract}

Primarily used software for the whole map-making process included ArcGIS Pro and Adobe Illustrator.

Data processing took an important part in this project. There was no attribute with coordinates to be used, however, the affected places were written in the attribute "Location". Naturally, each disaster covers a larger area of multiple places which are separated by commas in the mentioned attribute. Hence the idea was to divide multiple places of one disaster into its own separated records in order to use a geocoding feature in ArcGIS Pro.

With the prior data adjustment, the geocoding feature in ArcGIS Pro was used to assign a location to its place according to Location and Country attributes. $70 \%$ of records were matched automatically, the rest was either tied or unmatched. Those were assigned manually by choosing one of the suggested locations or picked directly from a map.

After having all disasters located on a map, a hexagon grid was generated with each hexagon covering $10,000 \mathrm{~km} 2$. The grid was then intersected with a feature layer of European countries which created the first hexagonal map of Europe. Some hexagons were adjusted with countries' borders overlay so that their shape and topology were the closest to reality and the map easily readable.

An Identity function was used to assign hexagon IDs to every point based on their overlay. Due to the data processing steps made, multiple points of the same disaster were located in the same hexagon leading to deceptive results. Delete Identical function was used to solve the problem by deleting duplicate records based on the disaster ID and hexagon ID. A new feature layer of hexagons containing at least one disaster was made by intersecting with the point feature layer of disasters.

To create a quantitative cartogram visualized by graduated colors based on disaster density, first, the summarize function was called to count the number of disasters located in each hexagon. Class intervals were created by the quantile method based on its exponential distribution.

A qualitative cartogram was realized by the outline color of each hexagon according to the most occurred disaster subgroup in the region. First, a table with a number of disasters of the same subgroup and another table with a count of the most occurred subgroup within each polygon were created by the summarize function. The two tables were joined by the hexagon ID attribute and narrowed down by query select on rows with the count and the maximum count attributes matching. This table was then joined to the hexagon feature layer of Europe and visualized by unique symbols.

A graduated symbol map of black hexagons was created to draw attention to the regions where people were affected by natural disasters the most. Once again, the summarize function was called to get a total number of affected people and a total number of deaths. These two attributes were then put into one attribute in a new field showing both affected and dead people together. The table was then joined to the hexagon feature layer of Europe and visualized by graduated symbols into 7 intervals made by the quantile method due to its exponential distribution.

In the new layout, a map field and a map legend were generated and the layout was exported in PDF format.

All the graphical work including a title, a legend, a scale, colophony, a graph, and other visual components were created in Adobe Illustrator. The graph shows classification by a disaster subgroup and a disaster type and their overall percentage share in Europe within the last decade. The criteria for disaster to enter the database were put in the final map and finally, three disasters with the most affected people and three disasters with the most deaths were pointed out by a line segment leading to the main catastrophe characteristics. The whole map is linked by the shape of a hexagon. 
Looking at the final map, it's obvious that Europe is generally the most afflicted by meteorological and hydrological disasters. Floods in Central Europe and Southeast Europe affect peoples' lives the most, however, the price for the cruelest and the most killing disaster goes to the extreme summer heats in West Europe. It is also interesting to point out, that Finland, Iceland, and Cyprus have according to the EM-DAT database not experienced any natural disasters in the last decade.

Generally speaking, a map is a great tool for data visualization. Compared to data tables, the interpretation of a map is much easier due to its added geographical information and the map itself is more attractive to observe. Moreover, the use of modern cartographic techniques provides a better understanding of a certain problem. 